ARTICLE

Received 13 Sep 2016 | Accepted 15 Apr 2017 | Published 7 Jun 2017 DOl: 10.1038/ncomms15640 OPEN

\title{
Ultra-bright and highly efficient inorganic based perovskite light-emitting diodes
}

Liuqi Zhang ${ }^{1}$, Xiaolei Yang ${ }^{1}$, Qi Jiang ${ }^{1}$, Pengyang Wang ${ }^{1}$, Zhigang Yin ${ }^{1,2}$, Xingwang Zhang ${ }^{1,2}$, Hairen Tan ${ }^{3}$, Yang (Michael) Yang ${ }^{4}$, Mingyang Wei ${ }^{3}$, Brandon R. Sutherland ${ }^{3}$, Edward H. Sargent ${ }^{3} \&$ Jingbi You ${ }^{1,2}$

Inorganic perovskites such as $\mathrm{CsPbX}_{3}(X=\mathrm{Cl}, \mathrm{Br}, \mathrm{I})$ have attracted attention due to their excellent thermal stability and high photoluminescence quantum efficiency. However, the electroluminescence quantum efficiency of their light-emitting diodes was $<1 \%$. We posited that this low efficiency was a result of high leakage current caused by poor perovskite morphology, high non-radiative recombination at interfaces and perovskite grain boundaries, and also charge injection imbalance. Here, we incorporated a small amount of methylammonium organic cation into the $\mathrm{CsPbBr}_{3}$ lattice and by depositing a hydrophilic and insulating polyvinyl pyrrolidine polymer atop the $\mathrm{ZnO}$ electron-injection layer to overcome these issues. As a result, we obtained light-emitting diodes exhibiting a high brightness of $91,000 \mathrm{cdm}^{-2}$ and a high external quantum efficiency of $10.4 \%$ using a mixed-cation perovskite $\mathrm{Cs}_{0.87} \mathrm{MA}_{0.13} \mathrm{PbBr}_{3}$ as the emitting layer. To the best of our knowledge, this is the brightest and most-efficient green perovskite light-emitting diodes reported to date.

\footnotetext{
${ }^{1}$ Key Laboratory of Semiconductor Materials Science, Institute of Semiconductors, Chinese Academy of Sciences, Beijing 100083, China. ${ }^{2}$ College of Materials Science and Opto-electronic Technology, University of Chinese Academy of Sciences, Beijing 100049, China. ${ }^{3}$ Department of Electrical and Computer Engineering, University of Toronto, 35 St George Street, Toronto, Ontario, Canada M5S 1A4. ${ }^{4}$ College of Optical Science and Engineering, Zhejiang University, Hangzhou 310027, China. Correspondence and requests for materials should be addressed to X.Z.(email: xwzhang@semi.ac.cn) or to J.Y. (email: jyou@semi.ac.cn).
} 
O rganic-inorganic perovskites have received extensive attention in recent years in view of their attractive electrical and optical properties. Solution-processed perovskite solar cells have demonstrated a high certified power conversion efficiency of $22.1 \%$, which is comparable to photovoltaics made from traditional inorganic semiconductor materials such as Si, CIGS and CdTe (refs 1-8). In addition, they have been utilized as efficient low-threshold gain media in optically pumped lasers ${ }^{9,10}$. Perovskite materials exhibit high photoluminescence quantum yield (PLQY, $>90 \%$ in solution for nanocrystals) and high colour purity with narrow emission linewidths $<20 \mathrm{~nm}$ (refs 11-15). These features make them promising candidates as new materials for light-emitting diodes (LEDs).

Electroluminescence (EL) from trihalide organic-inorganic perovskite-based LEDs (PeLEDs) was first reported in 2014 (ref. 16). The peak brightness of these LEDs was of order $300 \mathrm{~cd} \mathrm{~m}^{-2}$ at green wavelengths, and the external quantum efficiency (EQE) from $\mathrm{CH}_{3} \mathrm{NH}_{3} \mathrm{PbI}_{3-x} \mathrm{Cl}_{x}$ (754 $\mathrm{nm}$ emission, red) and $\mathrm{CH}_{3} \mathrm{NH}_{3} \mathrm{PbBr}_{3}-(517 \mathrm{~nm}$ emission, green $)$ based LEDs were 0.76 and $0.1 \%$, respectively ${ }^{16}$. By interface engineering and perovskite layer optimization, the EQE was increased to over 3\% (refs 17-27). A breakthrough in organic-inorganic PeLEDs was achieved by controlling the crystallization process of $\mathrm{CH}_{3} \mathrm{NH}_{3} \mathrm{PbBr}_{3}$ by adopting a nanocrystal pinning method. As a result, a dense film with small crystal domains $(<100 \mathrm{~nm})$ was obtained, effectively confining charge carriers. These devices demonstrated an impressive EQE of $8.53 \%$ (ref. 28). More recently, by confining electrons and holes to two-dimensional (2D) perovskites ${ }^{29}$, Yuan et al. and Wang et al. obtained near-infrared EQEs of 8.8 and $11.7 \%$, respectively ${ }^{30,31}$. Combined with nanocrystal pinning and $2 \mathrm{D}$ perovskites, Rand et al. also achieved close to $10 \% \mathrm{EQE}$ of organic-inorganic $\mathrm{LEDs}^{32}$

Compared with monovalent organic cation-based lead-halide perovskites, all inorganic perovskites exhibit improved thermal stability and more efficient PL, making them attractive in a number of optoelectronic device applications ${ }^{14,15,33,34}$. Inorganic perovskites such as $\mathrm{CsPbX}_{3}(\mathrm{X}=\mathrm{Cl}, \mathrm{Br}, \mathrm{I})$ have attracted great attention due to their improved thermal stability and higher PLQY in comparison with organic-cation perovskites. Recently, $\mathrm{CsPbX}_{3}$ nanocrystals have been successfully synthesized and used as emitting materials for LEDs ${ }^{14,15}$. $\mathrm{CsPbX}_{3}$ thin film-based LEDs have also been demonstrated ${ }^{34}$. However, the EQE of the LEDs based on these materials remain $<1 \%$ (refs 15,34). During revising of this manuscript, Li et al. and Ling et al. both reported inorganic $\mathrm{CsPbBr}_{3}$ LEDs with about 6\% EQE by surface passivation of perovskite nanocrystals and controlling perovskite thin film morphology, respectively ${ }^{35,36}$. There is still much more room for improvement in brightness and efficiency. The low efficiency may arise from high leakage current due to poor morphology (high density of pinholes), significant nonradiative recombination at the interface of perovskite/injection layers and within the perovskite layer itself and charge injection imbalance ${ }^{15,34}$.

In this report, we achieved high-quality dense $\mathrm{CsPbBr}$ perovskite thin films by incorporating a small amount of organic methylammonium cation into the lattice and by using a hydrophilic insulating polymer interface layer on top of the $\mathrm{ZnO}$ electron-injecting electrode. We fabricated high performance mixed-cation perovskite LEDs with an active layer composition of $\mathrm{Cs}_{0.87} \mathrm{MA}_{0.13} \mathrm{PbBr}_{3}$. These LEDs exhibited a peak brightness of $91,000 \mathrm{~cd} \mathrm{~m}^{-2}$ and a peak EQE of $10.43 \%$. This represents the brightest and most-efficient green perovskite LEDs reported to date $\mathrm{e}^{28,30,31}$.

\section{Results}

Morphology of $\mathrm{CsPbBr}_{3}$ films. $\mathrm{CsPbBr}_{3}$ perovskite thin films were fabricated by spin-coating a $\mathrm{CsBr}: \mathrm{PbBr}_{2}$ precursor from dimethyl sulfoxide (DMSO) onto substrates, followed by annealing at $100^{\circ} \mathrm{C}$ for $20 \mathrm{~min}$ to remove residual solvent and to induce perovskite crystallization. A high ratio of $\mathrm{CsBr}: \mathrm{PbBr}_{2}$ $(2.2: 1)$ precursor solution was used to guarantee the formation of pure phase $\mathrm{CsPbBr}_{3}$, while the excess $\mathrm{CsBr}$ would be readily precipitated during solution stirring ${ }^{33}$. As shown in Fig. 1a, the perovskite film directly deposited onto the electron-injection layer of $\mathrm{ZnO}$ exhibited a high density of pinholes. To improve the surface morphology, we first introduced a thin hydrophilic insulating polymer, polyvinyl pyrrolidine (PVP), between the $\mathrm{ZnO}$ and perovskite layers. The density of pinholes was largely reduced by inserting the PVP intermediate layer, as shown in Fig. 1b. Real-time contact angle results showed that PVP-modified $\mathrm{ZnO}$ films have increased hydrophilicity (Supplementary Fig. 1). As a result, the PVP-modified substrate has better wetting of the hydrophilic perovskite precursor solution, leading to uniform growth of perovskite films with reduced pinholes. Although the perovskite film surface coverage was significantly improved by introducing the PVP intermediate layer, there was still an appreciable density of pinholes. To improve further the morphology of the perovskite film, we added a small amount of $\mathrm{CH}_{3} \mathrm{NH}_{3} \mathrm{Br}$ (MABr) into the precursor solution. We hypothesized that molecular pinning would help reduce pinholes by better controlling the crystallization kinetics of the $\mathrm{CsPbBr}_{3}$ films $\mathrm{P}^{37,38}$. Figure 1c shows that the morphology of the perovskite film was improved considerably once we did add $\mathrm{MABr}$, leading to now a negligible density of pinholes, which could be good for reducing current leakage in LEDs.

Crystal and band structure of $\mathrm{CsPbBr}_{3}$ films. We characterized the crystal structure of $\mathrm{CsPbBr}_{3}$ films deposited on bare $\mathrm{ZnO}$ and $\mathrm{ZnO} / \mathrm{PVP}$ substrates, and on $\mathrm{ZnO} / \mathrm{PVP}$ with the $\mathrm{MABr}$ additive. $\mathrm{X}$-ray diffraction pattern of these three films were almost identical, with all crystallographic signatures matching that of the pure $\mathrm{CsPbBr}_{3}$ phase (Supplementary Fig. 2). The band structure of $\mathrm{CsPbBr}_{3}$ films with and without the $\mathrm{MABr}$ additive were determined using ultraviolet photoelectron spectroscopy (UPS; Supplementary Fig. 3, Supplementary Table 1) in combination with linear absorption measurements (Supplementary Fig. 4). The conduction and valence band relative to vacuum of $\mathrm{CsPbBr}_{3}$ with $\mathrm{MABr}$ are located at -3.37 and $-5.71 \mathrm{eV}$, respectively. This could form a good alignment with electron-injecting layer such as $\mathrm{ZnO}(-3.84 \mathrm{eV}$; Supplementary Fig. 5) and hole-injecting such as CBP $(-6.0 \mathrm{eV})^{39}$ (Fig. 3b).

Chemical states of $\mathrm{CsPbBr}_{3}$ films. We also carried out X-ray photoelectron spectroscopy (XPS) measurements on $\mathrm{CsPbBr}_{3}$ films with and without the MABr additive (Supplementary Figs 6 and 7). The $\mathrm{Pb} 4 \mathrm{f}$ core level from pure $\mathrm{CsPbBr}_{3}$ can be fit to four peaks (Supplementary Fig. 7). Two main peaks are located at $138.9 \mathrm{eV}\left(\mathrm{Pb} 4 f_{7 / 2}\right)$ and $142.8 \mathrm{eV}\left(\mathrm{Pb} \mathrm{4} f_{5 / 2}\right)$, which correspond to $\mathrm{Pb}-\mathrm{Br}$ bonding 28,40 . Two additional weaker peaks at 137.1 and $141.9 \mathrm{eV}$ can be attributed to $\mathrm{Pb}$ metallic states ${ }^{28,40}$. After incorporating $\mathrm{MABr}$ into the $\mathrm{CsPbr}_{3}$ lattice, only $\mathrm{Pb}-\mathrm{Br}$ peaks were found, indicating that $\mathrm{Pb}$ metallic states, which are known to function as non-radiative recombination centres ${ }^{28,40}$, have been suppressed.

Photoluminescence of $\mathrm{CsPbBr}_{3}$ films. We carried out steady-state $\mathrm{PL}$ on $\mathrm{CsPbBr}_{3}$ thin films deposited from different conditions (Fig. 2a). The $\mathrm{CsPbBr}_{3}$ films directly deposited on $\mathrm{ZnO}$ showed weak green emission at $524 \mathrm{~nm}$ with a full width at 

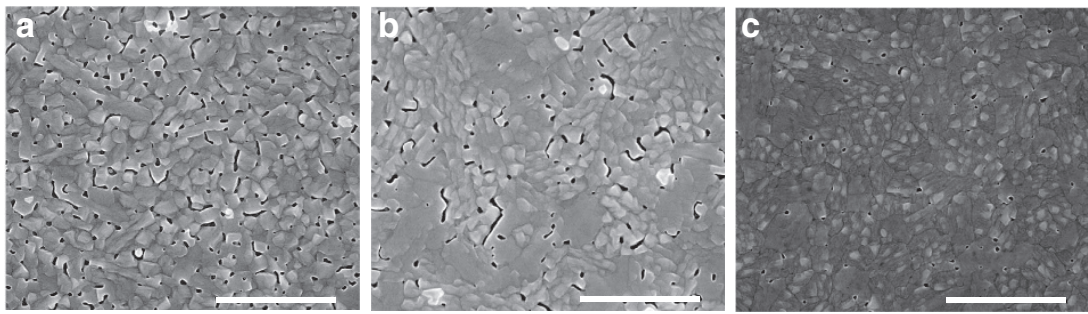

Figure 1 | Morphology of $\mathbf{C s P b B r}_{3}$ films deposited under different conditions. (a-c) Planar SEM images of $\mathrm{CsPbBr} 3$ deposited on $\mathrm{ZnO}, \mathrm{ZnO} / \mathrm{PVP}$ and $\mathrm{Cs}_{0.87} \mathrm{MA}_{0.13} \mathrm{PbBr}_{3}$ on $\mathrm{ZnO} / \mathrm{PVP}$, respectively, here PVP is polyvinyl pyrrolidine. The scale bar is $2 \mu \mathrm{m}$ in all images.

a

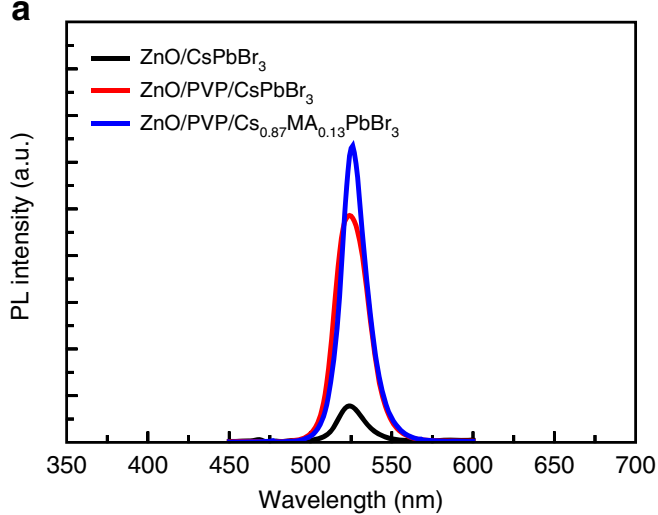

C

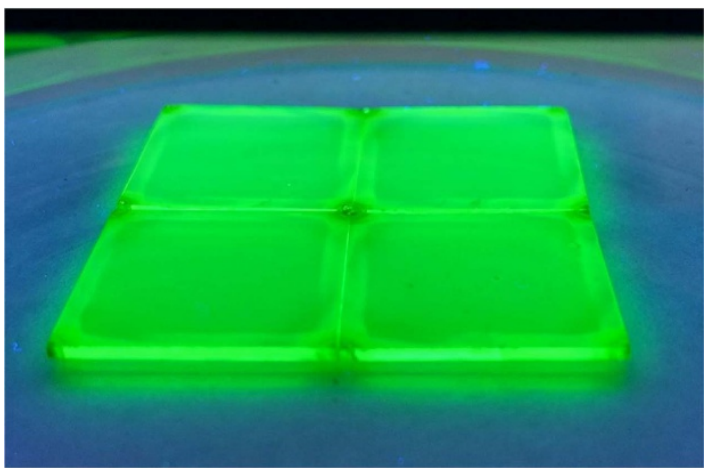

b

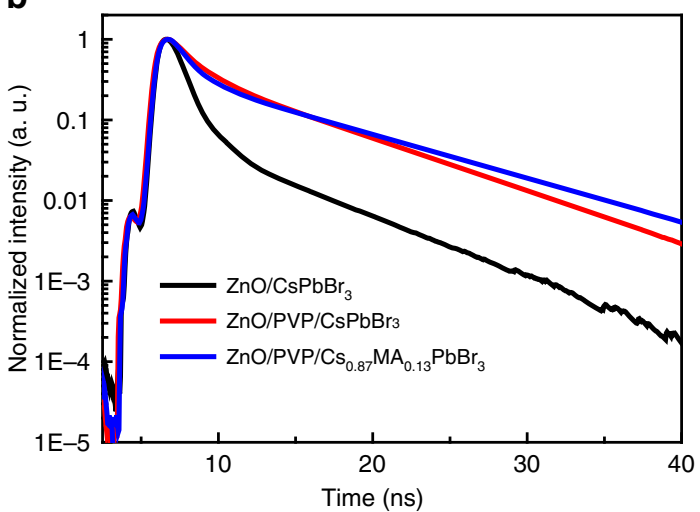

d

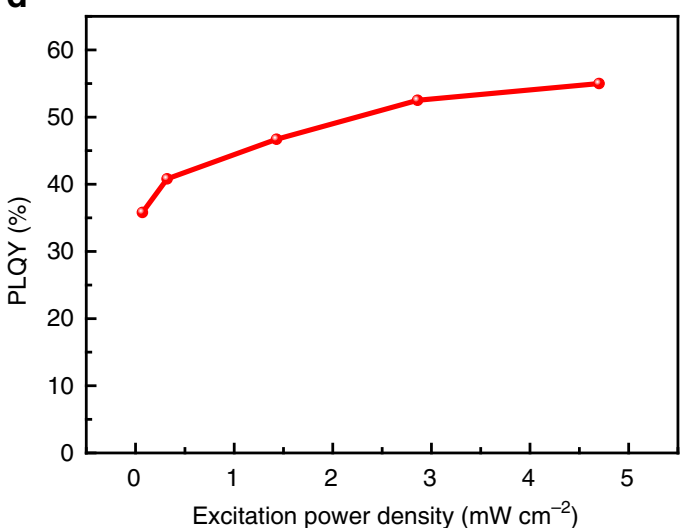

Figure 2 | PL behaviour of $\mathrm{CsPbBr}_{3}$ films deposited under different conditions. (a) Steady-state $\mathrm{PL}$ of $\mathrm{CsPbBr}$ films on $\mathrm{ZnO}, \mathrm{ZnO} / \mathrm{PVP}$ and $\mathrm{Cs}_{0.87} \mathrm{MA}_{0.13} \mathrm{PbBr}_{3}$ film on $\mathrm{ZnO} / \mathrm{PVP}$, respectively, here $\mathrm{PVP}$ is polyvinyl pyrrolidine, $\mathrm{MA}$ is $\mathrm{CH}_{3} \mathrm{NH}_{3}$. (b) Time-resolved $\mathrm{PL}$ of $\mathrm{CsPbBr}$ films on $\mathrm{ZnO}$ and $\mathrm{ZnO} / \mathrm{PVP}$ and $\mathrm{Cs}_{0.87} \mathrm{MA}_{0.13} \mathrm{PbBr}_{3}$ on $\mathrm{ZnO} / \mathrm{PVP}$. (c) PL image of $\mathrm{Cs}_{0.87} \mathrm{MA}_{0.13} \mathrm{PbBr}_{3}$ films on $\mathrm{ZnO} / \mathrm{PVP}$ under ultraviolet lamp excitation. (d) PLQY of $\mathrm{Cs}_{0.87} \mathrm{MA}_{0.13} \mathrm{PbBr}_{3}$ as a function of excitation power density.

half maximum (FWHM) of $24 \mathrm{~nm}$. Perovskites deposited onto the PVP-modified $\mathrm{ZnO}$ showed a dramatic increase in $\mathrm{PL}$ intensity, indicating that non-radiative recombination in the perovskite layer or at the interfaces has been significantly suppressed. The enhancement of PL is posited to arise from several phenomena. First, the improved morphology may reduce non-radiative recombination at grain boundaries (Fig. 1a,b), leading to enhanced PL. Second, PVP could passivate ZnO surface defects ${ }^{41}$, which could act as non-radiative recombination traps at the interface of $\mathrm{ZnO} /$ perovskite. After PVP modification, the PL emission intensity and carrier lifetime of $\mathrm{ZnO}$ increased considerably (Supplementary Fig. 8), indicating a reduced surface defects of $\mathrm{ZnO}$ with PVP layer. Similar enhancement were also observed while coating PVP on perovskite surface, further confirming our argument (Supplementary Fig. 8).
The PL was further improved by introducing $\mathrm{MABr}$ into the $\mathrm{CsPbBr}$ lattice. This increase in PL intensity is consistent with the reduction of perovskite grain boundaries, as well as the suppression of $\mathrm{Pb}$ metallic recombination centres, which were confirmed by scanning electron microscopy (SEM) and XPS results, respectively (Fig. 1c and Supplementary Fig. 7). The emission peak from $\mathrm{CsPBr}_{3}$ has been slightly shifted from 524 to $526 \mathrm{~nm}$ after adding $\mathrm{MABr}$, indicating that a small fraction of MA cations have been introduced into the $\mathrm{CsPbBr}_{3}$ crystal lattice. A single Gaussian emission peak at $526 \mathrm{~nm}$ shows that the $\mathrm{CsPbBr}_{3}$ layer with $\mathrm{MABr}$ additive is a pure perovskite phase, which could be ascribed to the formation of an alloy phase of $\mathrm{Cs}_{1-x} \mathrm{MA}_{x} \mathrm{PbBr}_{3}$. We estimate that the $\mathrm{MA}$ content in this alloyed perovskite is 0.13 , that is, $\mathrm{Cs}_{0.87} \mathrm{MA}_{0.13} \mathrm{PbBr}_{3}$-based on the band-edge emission as shown in Fig. $2 \mathrm{a}$, and the band-edge 


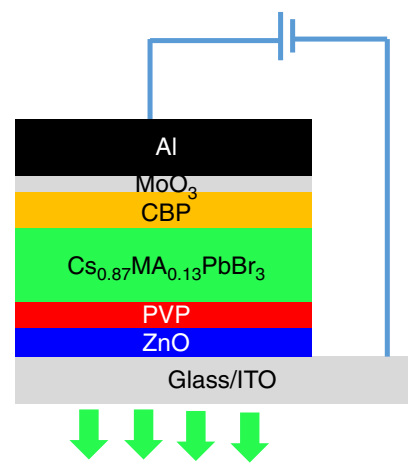

b

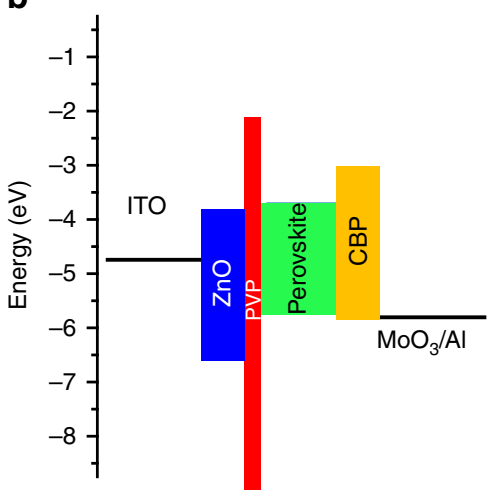

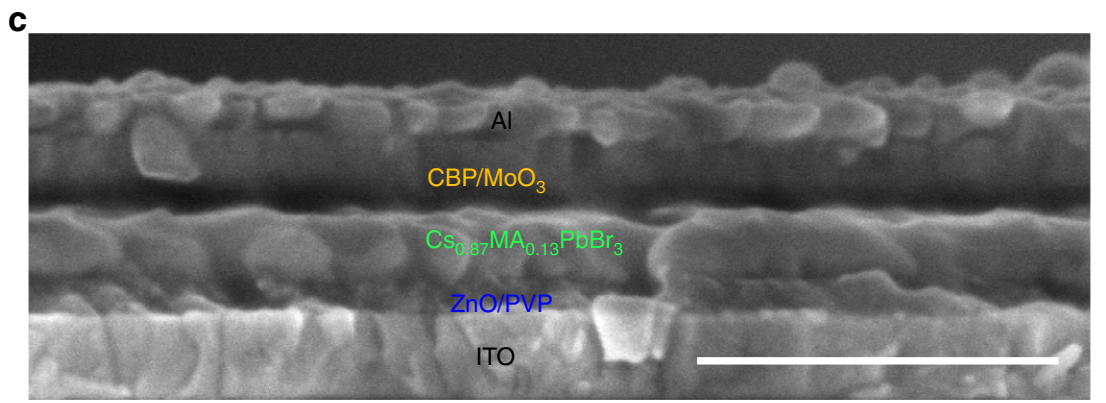

Figure 3 | Device structure of $\mathbf{C s P b B r}_{3}$ inorganic-based perovskite LEDs. (a) Device structure, glass/ITO/ZnO/PVP/CsPbBr $/ \mathrm{CBP}_{3} / \mathrm{MoO}_{3} / \mathrm{Al}$, here $\mathrm{PVP}$ is polyvinyl pyrrolidine, $\mathrm{CBP}$ is $4,4^{\prime}$ - $\mathrm{Bis}\left(\mathrm{N}\right.$-carbazolyl)-1, $1^{\prime}$-biphenyl. $\mathrm{ZnO}$ are $\mathrm{CBP} / \mathrm{MoO}_{3}$ are used as the electron and hole injection layers, respectively. $\mathrm{PVP}$ was used to improve peorvskite morphology and also passivate the interface defects and improve charge injection balance. (b) Band alignment of each functional layer. (c) Cross-sectional SEM image of the LEDs, scale bar is $500 \mathrm{~nm}$.

emission form pure $\mathrm{MAPbBr}_{3}(540 \mathrm{~nm})^{28}$ according to the linear relationship $\quad \mathrm{E}_{\mathrm{g}, \mathrm{Cs} 1-x \mathrm{MA} x \mathrm{PbBr} 3}=(1-x) \mathrm{E}_{\mathrm{g}, \mathrm{CsPbBr}}+x \mathrm{E}_{\mathrm{g}, \mathrm{MAPbBr} 3}$ (ref. 42). The final MABr content in $\mathrm{CsPbBr}_{3}$ as estimated from the change in bandgap is approximately consistent with the initial precursor composition where $\mathrm{CsBr}: \mathrm{PbBr}_{2}: \mathrm{MABr}=2.2: 1: 0.1$ and only $1 \mathrm{~mol} \mathrm{CsBr}$ contributes to the formation of $\mathrm{CsPbBr}_{3}$ and the initial $\mathrm{MABr}$ ratio is $0.1 \mathrm{~mol}$. The linear absorption of $\mathrm{CsPbBr}_{3}$ with and without $\mathrm{MABr}$ was consistent with the PL results (Supplementary Fig. 4). We further observed that the PL FWHM narrowed from $24 \mathrm{~nm}$ to $18 \mathrm{~nm}$ after introducing MABr. This indicates that the $\mathrm{MABr}$ additive has improved the sharpness of the perovskite band edge.

We next acquired time-resolved PL decay spectra of the different perovskite layers (Fig. 2b). The time-resolved PL curves were fit to bi-exponential decays, where the fast decay component is associated with trap-assisted recombination at grain boundaries or surfaces, and the slow decay is ascribed to radiative recombination inside the bulk perovskite phase ${ }^{28,43}$. For the $\mathrm{ZnO} / \mathrm{CsPbBr}{ }_{3}, \mathrm{PVP} / \mathrm{CsPbBr}$ and $\mathrm{PVP} / \mathrm{Cs}_{0.87} \mathrm{MA}_{0.13} \mathrm{PbBr}_{3}$ films, the decay times are $\left(\tau_{1}=1.2 \mathrm{~ns}, \tau_{2}=4.6 \mathrm{~ns}\right), \quad\left(\tau_{1}=2.1 \mathrm{~ns}\right.$, $\left.\tau_{2}=6.4 \mathrm{~ns}\right)$ and $\left(\tau_{1}=1.8 \mathrm{~ns}, \quad \tau_{2}=7.5 \mathrm{~ns}\right)$, respectively. Generally, it was found that the PL lifetime of the perovskite film is increased after PVP modification, and further increased after the addition of MABr. We observed that the Cs-MA mixed perovskite has a marginally faster decay component in comparison with pure Cs perovskite. We hypothesize that this may be a result of increased surface defects in the Cs-MA mixed perovskite. However, the slow decay component of Cs-MA exhibited a longer lifetime, indicative of less bulk defects, consistent with the observed reduction of $\mathrm{Pb}$ metallic states (Supplementary Fig. 7). Although Cs-MA sample showed shorter lifetime in fast decay component compared with pure Cs, stronger PL from Cs-MA samples (Fig. 3a) indicated that the overall defects including surface and bulk defects in Cs-MA are less than that of in pure Cs. The $\mathrm{Cs}_{0.87} \mathrm{MA}_{0.13} \mathrm{PbBr}_{3}$ films show bright and uniform green PL under ultraviolet lamp excitation (Fig. 2c). Both PVP interface engineering and MABr lattice incorporation enhanced the PL emission of the perovskite film, which is beneficial to realize high performance LEDs.

The PLQY of $\mathrm{Cs}_{0.87} \mathrm{MA}_{0.13} \mathrm{PbBr}_{3}$ was measured as a function of excitation power density (Fig. 2d). As seen in other perovskites, the PLQY increases with excitation power ${ }^{30,31}$. This is attributed to state-filling of recombination centres in the perovskite layer ${ }^{30,31}$. Our inorganic-based perovskite materials, $\mathrm{Cs}_{0.87} \mathrm{MA}_{0.13} \mathrm{PbBr}_{3}$, exhibited high quantum yield (35.8\%) even at low light intensity $\left(0.07 \mathrm{~mW} \mathrm{~cm}{ }^{-2}\right)$. This is significantly higher than previous reports at a similar order of power excitation, indicative of reduced non-radiative recombination centres in the perovskite laye ${ }^{30,31}$. Upon increasing the excitation intensity to $4.70 \mathrm{~mW} \mathrm{~cm}^{-2}$, the quantum efficiency increased to as high as $55 \%$. The high PL quantum yield suggests promise for high EQE LEDs.

Light-emitting diodes based on $\mathrm{CsPbBr}_{3}$ films. We fabricated LEDs consisting of glass/indium tin oxide (ITO)/ZnO/PVP/ $\mathrm{CsPbBr}_{3} / \mathrm{CBP} / \mathrm{MoO}_{3} / \mathrm{Al}$ (Fig. 3a), where $\mathrm{CsPbBr}_{3}$ is the emitting layer, Apart from ITO and $\mathrm{MoO}_{3} / \mathrm{Al}$, which were deposited in vacuum, all layers were solution processed via spin coating. The band alignment of the $\mathrm{CsPbBr}_{3}$ LEDs could be drawn as shown in Fig. $3 \mathrm{~b}$ based on the band structure of $\mathrm{CsPbBr}_{3}$ and $\mathrm{ZnO}$ (Supplementary Figs 3 and 5), and also the valence band of CBP $(-6.0 \mathrm{eV})^{39}$. $\mathrm{ZnO}$ and $\mathrm{CBP} / \mathrm{MoO}_{3}$ are used as the electron and hole injection layers, respectively. In addition to improve perovskite morphology and also passivate the interface defects, which has been illustrate above (Figs 1 and 2). PVP layer could also induce an electron-injection barrier (Fig. 3b), which could improve charge injection balance, this will be discussed later. The 
a

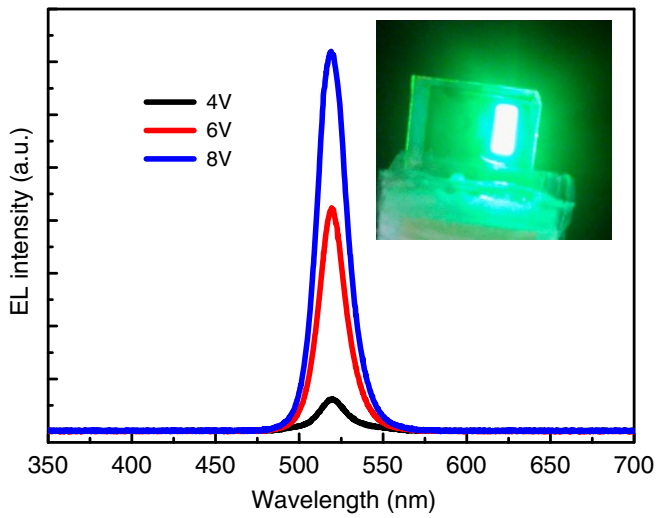

c

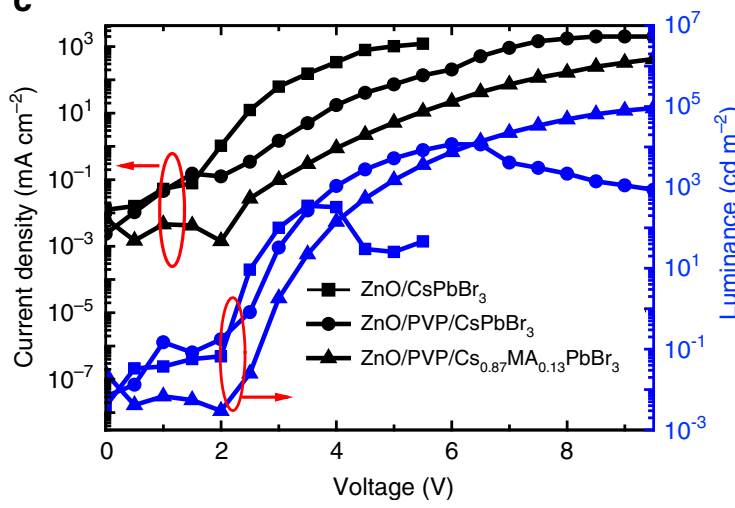

b

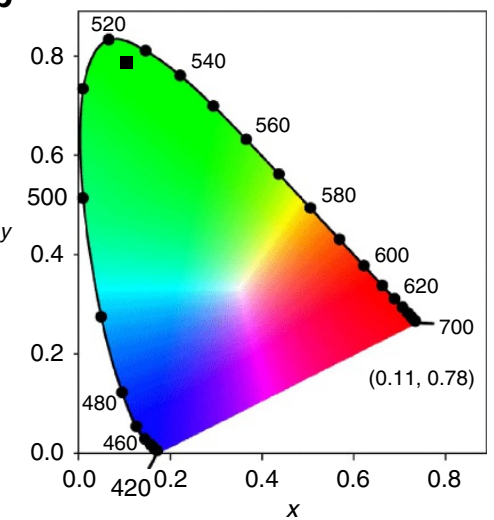

d

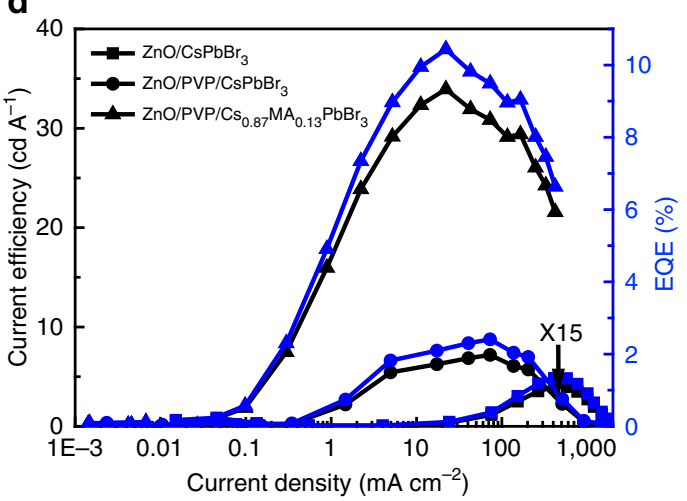

Figure 4 | EL performance of the devices. (a) EL spectra of $\mathrm{Cs}_{0.87} \mathrm{MA}_{0.13} \mathrm{PbBr}_{3}$-based devices under varying voltage bias (the emission image is shown in inset). (b) The corresponding CIE coordinate. (c) $I-V$ and voltage-light intensity ( $L-V$ ) curves for the devices with and without PVP buffer layer or with and without $\mathrm{CH}_{3} \mathrm{NH}_{3} \mathrm{Br}(\mathrm{MABr})$ additive, that is, $\mathrm{ZnO} / \mathrm{CsPbBr}_{3}, \mathrm{ZnO} / \mathrm{PVP} / \mathrm{CsPbBr}$ and $\mathrm{ZnO} / \mathrm{PVP} / \mathrm{Cs}_{0.87} \mathrm{MA} \mathrm{A}_{0.13} \mathrm{PbBr}{ }_{3}$, respectively, here $\mathrm{PVP}$ is polyvinyl pyrrolidine. (d) Current efficiency and EQE of devices with and without $\mathrm{PVP}$ buffer layer, with and without $\mathrm{CH}_{3} \mathrm{NH} \mathrm{H}_{3} \mathrm{Br}(\mathrm{MABr})$ additive, that is,

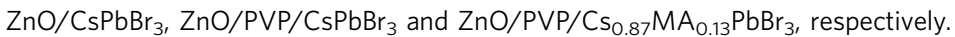

electrons and holes injected from each side recombine radiatively in the perovskite layer, resulting in photon emission. A crosssectional SEM image of a typical device showed a clear sandwich structure (Fig. 3c). The thicknesses of the $\mathrm{ZnO} / \mathrm{PVP}, \mathrm{CsPbBr}_{3}$ and $4,4^{\prime}$-Bis(N-carbazolyl)-1, $1^{\prime}$-biphenyl (CBP)/ $\mathrm{MoO}_{3}$ layers are $\sim 45 \mathrm{~nm}, 100 \mathrm{~nm}$ and $80 \mathrm{~nm}$, respectively.

Electroluminescence of light-emitting diodes. The EL spectra of $\mathrm{CsPbBr}{ }_{3}$ and $\mathrm{Cs}_{0.87} \mathrm{MA}_{0.13} \mathrm{PbBr}_{3}$-based devices are centred at 516 and $520 \mathrm{~nm}$, respectively (Supplementary Fig. 9). Compared to the PL emission at $526 \mathrm{~nm}$ for $\mathrm{Cs}_{0.87} \mathrm{MA}_{0.13} \mathrm{PbBr}_{3}$, the EL emission showed a slight blue shift to $520 \mathrm{~nm}$, which has also been observed in other perovskite-based LEDs ${ }^{16,44}$. The blue shift in the EL spectrum could be ascribed to free carrier emission, as already demonstrated in several perovskite systems ${ }^{16,44,45}$. For the devices using $\mathrm{Cs}_{0.87} \mathrm{MA}_{0.13} \mathrm{PbBr}_{3}$ as an emitting layer, the EL spectrum as a function of voltage bias was measured (Fig. $4 \mathrm{a}$ ), and an EL image of the device under operation was taken (inset of Fig. 4a). The EL showed very narrow emission $(F W H M=18 \mathrm{~nm})$ and high colour purity. This spectral line width is narrower than that of previously reported perovskite nanocrystal-based LEDs $^{14,15}$. The devices exhibited saturated and pure colour (90.2\%) at green wavelengths, with Commission Internationale de l'Eclairage (CIE) chromaticity coordinates at $(0.11,0.78)$ (Fig. $4 \mathrm{~b})$.

We measured the voltage-current $(I-V)$ curve of the devices (Fig. 4c) and found that the control devices (without both PVP and $\mathrm{MABr}$ ) showed higher injection current. This could be two reasons: one is high density of pinholes in the perovskite layer which results in a significant electron and hole injection leakage; and another could be the imbalanced charge injection. After introducing the PVP buffer layer and the MABr additive, the injection current of $\mathrm{CsPbBr}_{3}$ device was significantly reduced, indicating that the current leakage and charge injection imbalance has been suppressed. The turn-on voltage was slightly increased after inserting an immediate layer of PVP, which could be mainly due to the injection barrier caused by the insulating nature of PVP layer ${ }^{46}$. The further minor increase of turn-on voltage after $\mathrm{MABr}$ incorporation might be ascribed to deeper valence band of $\mathrm{Cs}_{0.87} \mathrm{MA}_{0.13} \mathrm{PbBr}_{3}(5.71 \mathrm{eV})$ compared to $\mathrm{Cs} \mathrm{PbBr}_{3}$ $(5.50 \mathrm{eV})$ (Supplementary Fig. 3, Supplementary Table 1$)^{47}$. Similar phenomenon has also been found by Sun et al. in FA-Cs mixture nanocrystal-based LEDs ${ }^{48}$. Although the turn-on voltage increased after incorporating PVP buffer layer and adding $\mathrm{MABr}$ salt, it can be calculated that the current efficiency are significantly increased from $0.02 \mathrm{~cd} \mathrm{~A}^{-1}$ $(\mathrm{CsPbBr} 3)$ to $1 \mathrm{~cd} \mathrm{~A}^{-1} \quad\left(\mathrm{PVP} / \mathrm{Cs}_{0.87} \mathrm{MA}_{0.13} \mathrm{PbBr}_{3}\right)$ at small brightness $\left(1 \mathrm{~cd} \mathrm{~m}^{-2}\right)$. The increase of current efficiency indicated the non-radiative recombination has been suppressed, which will be discussed later. The control devices showed a maximum brightness of $300 \mathrm{~cd} \mathrm{~m}^{-2}$ (Fig. 4c). The maximum brightness was dramatically increased to $11600 \mathrm{~cd} \mathrm{~m}^{-2}$ by introducing the PVP intermediate layer. Consistent with this was an observed increase in the current efficiency from $0.26 \mathrm{~cd} \mathrm{~A}^{-1}$ to $7.19 \mathrm{~cd} \mathrm{~A}^{-1}$ (Fig. 4d). The EQE of the LEDs from control devices of $\mathrm{CsPbBr}_{3}$ on $\mathrm{ZnO}$ is $<0.1 \%$. The addition of the PVP buffer layer improved the quantum efficiency to $2.4 \%$ (Fig. 4d). 
Table 1 | Device performance with and without PVP intermediate layers or $\mathrm{CH}_{3} \mathrm{NH}_{3} \mathrm{Br}(\mathrm{MABr})$ additive.

\begin{tabular}{|c|c|c|c|c|}
\hline Devices & $v_{\text {th }}(V)$ & $L_{\max }\left(\mathrm{cd} \mathrm{m}^{-2}\right)$ & $\begin{array}{c}\text { Current efficiency } \\
\left(\mathrm{Cd} \mathrm{A}^{-1}\right)\end{array}$ & EQE (\%) \\
\hline $\begin{array}{l}\mathrm{ZnO} / \mathrm{CsPbBr}_{3} \\
\mathrm{ZnO} / \mathrm{PVP} / \mathrm{CsPbBr}_{3} \\
\mathrm{ZnO} / \mathrm{PVP} / \mathrm{Cs}_{0.87} \mathrm{MA}_{0.13} \mathrm{PbBr}_{3}\end{array}$ & $\begin{array}{l}2.3 \\
2.6 \\
2.9\end{array}$ & $\begin{array}{c}350 \\
11,600 \\
91,000\end{array}$ & $\begin{array}{l}0.26 \\
7.19 \\
33.9\end{array}$ & $\begin{array}{c}0.09 \\
2.41 \\
10.43\end{array}$ \\
\hline
\end{tabular}

The MABr salt additive further improves both the maximum brightness and EQE. The maximum brightness of $\mathrm{Cs}_{0.87} \mathrm{MA}_{0.13} \mathrm{PbBr}_{3}$-based LEDs increased to as high as $91,000 \mathrm{~cd} \mathrm{~m}^{-2}$. The current efficiency and quantum efficiency were increased to $33.9 \mathrm{~cd} \mathrm{~A}^{-1}$ and $10.43 \%$, respectively (Fig. 4d). The best devices exhibited an internal quantum efficiency (IQE) of $47 \%$ calculated by IQE $=2 n^{2} \mathrm{EQE}$, where $n$ is the refractive of glass $(1.5)^{31}$. The device performance parameters are summarized in Table 1. To the best of our best knowledge, these devices are the brightest and most-efficient perovskite-based LEDs emitting at green wavelengths reported to date.

These devices also demonstrated high reproducibility (Supplementary Fig. 10). The high performance in the $\mathrm{ZnO} / \mathrm{PVP} / \mathrm{Cs}_{0.87} \mathrm{MA}_{0.13} \mathrm{PbBr}_{3}$ LED is a result of careful optimization of the interfaces and perovskite layers (Supplementary Figs 11-13, Supplementary Tables 2 and 3). It was found that a high amount of MABr compositionally mixed with $\mathrm{CsPbBr}_{3}$ led to a significant decrease in LED performance. We attribute this to poor morphology (Supplementary Fig. 14).

We tested the stability of $\mathrm{CsPbBr}_{3}$ LEDs (Supplementary Fig. 15). Consistent with what was observed in previous perovskite LED reports ${ }^{33,49}$, the devices decayed after several minutes. The decay mechanism is hypothesized to be a result of ion migration under steady-state voltage bias. To improve device stability, we attempted to incorporate ion-migration-inhibiting polymers ${ }^{50}$, such as PVP, into the perovskite layer. The results indeed showed that the stability had been significantly improved-the output was stable for several hours. While encouraging, the efficiency of this polymer-blended device is reduced in comparison with the $\mathrm{ZnO} / \mathrm{PVP} / \mathrm{Cs}_{0.87} \mathrm{MA}_{0.13} \mathrm{PbBr}_{3}$ devices (Supplementary Fig. 16). We will study in more depth the underlying phenomena leading to this stability/efficiency compromise in our future work. We have also tested the transient light emission response of our perovskite LEDs (Supplementary Fig. 17). Nearly instantaneous turn-on was achieved with a response time about $18 \mathrm{~ms}$ to reach their maximum output light intensities. Such a fast turn-on is comparable to that of conventional LEDs ${ }^{51}$.

\section{Discussion}

We found that the improvement via PVP could be due to three reasons. First, the improvement of the perovskite film morphology, as shown in Fig. 1a,b, leads to reduced pinholes which minimize current leakage (Fig. 4a). Second, the suppression of non-radiative recombination at the $\mathrm{ZnO} /$ perovskite interface, which has been confirmed by PL results (Fig. 2a, Supplementary Fig. 8), improves the radiative efficiency.

In addition to improvement of perovskite film morphology and passivation of defects at interface, PVP could improve the charge injection balance in our perovskite LEDs, and thus enhancing devices EL efficiency. Similar mechanism has been proposed in previous reports, while using PMMA insulting layer in quantum dot LEDs ${ }^{46}$. It was found that the injection current from electron only devices is much higher than that of the current from hole only devices, while using $\mathrm{ZnO}$ and $\mathrm{CBP}$ as the main injection layers, respectively (Supplementary Fig. 18). These results indicated that the electron injected by $\mathrm{ZnO}$ is faster than holes injected by CBP, which could be due to the different carrier mobility of $\mathrm{ZnO}$ and $\mathrm{CBP}$ (ref. 46). This will lead to charge injection imbalance and also the excess electron current while using these layer as electron- and hole-injection layers in LEDs, and thus degrading EL efficiency ${ }^{46}$. Insulting PVP layer can slow down electron-injection via an energy barrier (Fig. 3b, Supplementary Fig. 18), an improvement of charge balance could be anticipated by inserting a PVP layer on $\mathrm{ZnO}$ surface as the electron-injection layer. In fact, the reduction of device injection current via PVP insertion confirmed the improvement of charge injection balance (Fig. $4 \mathrm{c})^{46}$, and thus improving EL emission efficiency.

There could be two key improvements of leading to superior performance for MA-Cs mixed perovskite devices. The first one is ascribed to the suppression of non-radiative recombination centres by eliminating the $\mathrm{Pb}$ metallic phase by compositionally blending $\mathrm{CsPBBr}_{3}$ with $\mathrm{MABr}$ to form the compound $\mathrm{Cs}_{0.87} \mathrm{MA}_{0.13} \mathrm{PbBr}_{3}$. PL (Fig. 2a) and time-resolved PL (Fig. 2b) both indicated that the less defects in Cs-MA films. Second, the key advance that led to the dramatic improvement in EL brightness and efficiency is the reduction of leakage current via improved morphology as a result of both PVP-modified $\mathrm{ZnO}$ and the MABr additive (Fig. 1c).

In summary, we have obtained high-quality $\mathrm{Cs}_{0.87} \mathrm{MA}_{0.13} \mathrm{PbBr}_{3}$ perovskite light-emitting thin films with minimized pinholes through electron-injecting interface passivation and perovskite composition modulation. These strategies jointly reduced the device leakage current. Furthermore, the non-radiative recombination centres at the interfaces and in the perovskite film were suppressed and also the charge injection balance were improved. As a result of these advances, we obtained ultra-bright and highly efficient inorganic perovskite-based LEDs. With additional optimizations to the perovskite and interfacial layers, the inorganic perovskite-based LEDs have promise to reach $20 \%$ EQE, making them competitive with materials such as semiconducting organics and colloidal quantum dots.

\section{Methods}

Preparation of perovskite solution and $\mathbf{Z n O}$ nanoparticles. $\mathrm{CsBr}$ (Sigma Aldrich, 99.9\%) and $\mathrm{PbBr}_{2}$ (Aldrich, 99.99\%) (CsBr: $\mathrm{PbBr}_{2}$ molar ratio of 2.2:1) solutions were prepared using DMSO as a solvent. The solution concentration is $0.5 \mathrm{M}$ (CsBr 1.1 M, $\left.\mathrm{PbBr}_{2} 0.5 \mathrm{M}\right)$. A high ratio of $\mathrm{CsBr}: \mathrm{PbBr}_{2}$ was used to suppress the formation of non-CsPbBr 3 phases. For films which incorporated the $\mathrm{CH}_{3} \mathrm{NH}_{3} \mathrm{Br}$ additive, $0.05 \mathrm{M} \mathrm{CH}_{3} \mathrm{NH}_{3} \mathrm{Br}$ was added to the solution $\left(\mathrm{CsBr}: \mathrm{PbBr}_{2}: \mathrm{MABr}=2.2: 1: 0.1\right)$. Comparative studies used additional ratios of $\mathrm{CsPbBr}{ }_{3}$ solutions, such as $\mathrm{CsBr}: \mathrm{PbBr}_{2}: \mathrm{MABr}=2: 1: 0.1,2.1: 1: 0.1,2.2: 1: 0.1,2.4: 1: 0.1$ and 2.2:1:0.2. The precursor solutions were stirred at $45^{\circ} \mathrm{C}$ overnight. And then the solution was stand for $4 \mathrm{~h}$ at room temperature, precipitates were formed in the CsBr-rich solution, top transparent solution was decanted and filtered for using. The details of precursor preparation procedures were shown in Supplementary Fig. 19. The $\mathrm{ZnO}$ nanoparticles were synthesized using a previously developed method $^{8}$. The synthesized $\mathrm{ZnO}$ nanoparticles were dispersed in methanol and n-butanol to form a $2 \% \mathrm{ZnO}$ nanoparticle solution.

Light-emitting diode fabrication. The glass/ITO substrate was sequentially washed with isopropanol, acetone, distilled water and isopropanol. The sheet resistance of ITO is $15 \Omega$ per square. $\mathrm{ZnO}$ nanoparticles of concentration $2 \%$ by weight were spin-coated onto ITO substrates at 2,000 r.p.m. for $30 \mathrm{~s}$ and then annealed at $150{ }^{\circ} \mathrm{C}$ for $15 \mathrm{~min}$. For control devices, perovskite precursor

( $\left.\mathrm{CsBr}: \mathrm{PbBr}_{2}=2.2: 1\right)$ was spin-coated onto $\mathrm{ZnO}$ at 2,000 r.p.m. for $2 \mathrm{~min}$, and then annealed at 100 for $20 \mathrm{~min}$. After, a $2 \mathrm{wt} \%$ CBP solution was spin-coated onto the perovskite. The devices were transferred into a vacuum chamber for $\mathrm{MoO}_{3} / \mathrm{Al}$ deposition. For PVP interface-modified devices, $0.5 \mathrm{wt} \%$ PVP solution in DMSO was spin-coated onto $\mathrm{ZnO}$ at 2,000 r.p.m. for $1 \mathrm{~min}$, and then annealed at $150{ }^{\circ} \mathrm{C}$ for 15 min to induce crosslinking. However, we found that the PVP is slightly washed away during spin-coating of the DMSO solution. The PVP thickness before and after DMSO solution washing are 8.4 and $5.0 \mathrm{~nm}$, respectively, which were measured by ellipsometer (Supplementary Fig. 20). For MABr additive devices, the ratio of $\mathrm{CsBr}: \mathrm{PbBr}_{2}: \mathrm{MABr}$ is 2.2:1:0.1, the final composition is $\mathrm{Cs}_{0.87} \mathrm{MA}_{0.13} \mathrm{PbBr}_{3}$ 
as determined by band-edge emission measurements. The solution concentration was $0.5 \mathrm{M}$. The device active area was $0.108 \mathrm{~cm}^{2}$.

\section{Materials and device characterization. A field emission SEM (FEI}

NanoSEM650) was used to acquire SEM images. The instrument uses an electron beam accelerated at $500 \mathrm{~V}$ to $30 \mathrm{kV}$, enabling operation at a variety of currents. Absorption measurement were carried out by Hitachi ultraviolet-visible U-4100 spectrophotometer. Absorbance was determined from a transmittance measurement using integrated sphere. PL measurements were carried out by FLS980 Spectrometer. The X-ray diffraction patterns $(\theta-2 \theta$ scans) were taken on a Rigaku D/MAX-2500 system using Cu k $\alpha(\lambda=1.5405 \AA)$ as the X-ray source. Scans were taken with $0.5 \mathrm{~mm}$ wide source and detector slits, and X-ray generator settings at $40 \mathrm{kV}$ and $30 \mathrm{~mA}$. XPS was performed on the Thermo Scientific ESCALab 250Xi using $200 \mathrm{~W}$ monochromated $\mathrm{Al} \mathrm{K \alpha}(1,486.6 \mathrm{eV})$ radiation. The $500 \mu \mathrm{m} \mathrm{X}$-ray spot was used for XPS analysis. The base pressure in the analysis chamber was $\sim 3 \times 10^{-10}$ mbar. Typically the hydrocarbon C1s line at $284.8 \mathrm{eV}$ from adventitious carbon is used for energy referencing. UPS samples were analyzed on a Thermo Scientific ESCALab 250Xi. The gas discharge lamp was used for UPS, with helium gas admitted and the HeI $(21.22 \mathrm{eV})$ emission line employed. The helium pressure in the chamber during analysis was $\sim 2 \mathrm{E}-8 \mathrm{mbar}$. The data was acquired with a $-10 \mathrm{~V}$ bias. The work function of the measured sample can be calculated from following equation: $h v-\phi=E_{\mathrm{Fermi}}-E_{\text {cutoff, }}$, here, $h v=21.22 \mathrm{eV}$, $E_{\mathrm{Fermi}}=21.08 \mathrm{eV}$ (using $\mathrm{Ni}$ as the standard sample for calibration), $E_{\text {cut-off }}$ is the cut-off shown in the corresponding Figures. The PLQY was measured using a Horiba Fluorolog system equipped with a single grating and a Quanta-Phil integration sphere coupled to the Fluorolog system with optic fibre bundles ${ }^{30}$. The following settings were applied for PLQY measurements: an excitation wavelength of $400 \mathrm{~nm}$; bandpass values of 10 and $5 \mathrm{~nm}$ for the excitation and emission slits, respectively; step increments of $1 \mathrm{~nm}$ and integration time of $0.5 \mathrm{~s}$ per data point. The excitation power density in the power-dependent PLQY characterization was tuned by varying the slit width on the Fluorolog monochromator. $J-V$ characteristics of LEDs were taken using a Keithley 2,400 source metre. Two Keithley 2,400 source metre units linked to a calibrated silicon photodiode were used to measure the current-voltage-brightness characteristics. The measurement system has been carefully calibrated by efficient InGaN/GaN LEDs with a similar photon response by PR-650 SpectraScan. A Lambertian profile was assumed in the calculation of $\mathrm{EQE}^{28,30,31}$

Data availability. The data that support the findings of this study are available from the corresponding author upon reasonable request.

\section{References}

1. Kojima, A., Teshima, K., Shirai, Y. \& Miyasaka, T. Organometal halide perovskites as visible-light sensitizers for photovoltaic cells. J. Am. Chem. Soc. 131, 6050-6051 (2009).

2. Kim, H. S. et al. Lead iodide perovskite sensitized all-solid-state submicron thin film mesoscopic solar cell with efficiency exceeding 9\%. Sci. Rep. 2, 591 (2012).

3. Lee, M. M., Teuscher, J., Miyasaka, T., Murakami, T. N. \& Snaith, H. J. Efficient hybrid solar cells based on meso-superstructured organometal halide perovskites. Science 338, 643-647 (2012).

4. Burschka, J. et al. Sequential deposition as a route to high-performance perovskite-sensitized solar cells. Nature 499, 316-319 (2013).

5. Jeon, N. J. et al. Solvent engineering for high-performance inorganic-organic hybrid perovskite solar cells. Nat. Mater. 13, 897-903 (2014).

6. Yang, W. S. et al. High-performance photovoltaic perovskite layers fabricated through intramolecular exchange. Science 348, 1234-1237 (2015).

7. Bi, D. et al. Efficient luminescent solar cells based on tailored mixed-cation perovskites. Sci. Adv. 2, e1501170 (2016).

8. You, J. et al. Improved air stability of perovskite solar cells via solutionprocessed metal oxide transport layers. Nat. Nanotechnol. 11, 75-81 (2016).

9. Xing, G. et al. Low-temperature solution-processed wavelength-tunable perovskites for lasing. Nat. Mater. 13, 476-480 (2014).

10. Zhu, H. et al. Lead halide perovskite nanowire lasers with low lasing thresholds and high quality factors. Nat. Mater. 14, 636-643 (2015).

11. Stranks, S. D. \& Snaith, H. J. Metal-halide perovskites for photovoltaic and light-emitting devices. Nat. Nanotechnol. 10, 391-402 (2015).

12. Deschler, F. et al. High photoluminescence efficiency and optically pumped lasing in solution-processed mixed halide perovskite semiconductors. J. Phys. Chem. Lett. 5, 1421-1426 (2014).

13. Zhang, F. et al. Brightly luminescent and color-tunable colloidal $\mathrm{CH}_{3} \mathrm{NH}_{3} \mathrm{PbX}_{3}$ $(\mathrm{X}=\mathrm{Br}, \mathrm{I}, \mathrm{Cl})$ quantum dots: potential alternatives for display technology. ACS Nano 9, 4533-4542 (2015).

14. Protesescu, L. et al. Nanocrystals of cesium lead halide perovskites $\left(\mathrm{CsPbX}_{3}\right.$, $\mathrm{X}=\mathrm{Cl}, \mathrm{Br}$, and I): novel optoelectronic materials showing bright emission with wide color gamut. Nano Lett. 15, 3692-3696 (2015).

15. Song, J. et al. Quantum dot light-emitting diodes based on inorganic perovskite cesium lead halides $\left(\mathrm{CsPbX}_{3}\right)$. Adv. Mater. 27, 7162-7167 (2015).
16. Tan, Z. K. et al. Bright light- emitting diodes based on organometal halide perovskite. Nat. Nanotechnol. 9, 687-692 (2014).

17. Li, G. et al. Efficient light-emitting diodes based on nanocrystalline perovskite in a dielectric polymer matrix. Nano Lett. 15, 2640-2644 (2015).

18. Jaramillo-Quintero, O. A., Sanchez, R. S., Rincon, M. \& Mora-Sero, I. Bright visible-infrared light emitting diodes based on hybrid halide perovskite with Spiro-OMeTAD as a hole-injecting layer. J. Phys. Chem. Lett. 6, 1883-1890 (2015).

19. Sadhanala, A. et al. Blue-green color tunable solution processable organolead chloride - bromide mixed halide perovskites for optoelectronic applications. Nano Lett. 15, 6095-6101 (2015).

20. Gil-Escrig, L. et al. Efficient photovoltaic and electroluminescent perovskite devices. Chem. Commun. 51, 569-571 (2015).

21. Li, J., Bade, S. G. R., Shan, X. \& Yu, Z. Single-layer light-emitting diodes using organometal halide perovskite/poly(ethylene oxide) composite thin films. Adv. Mater. 27, 5196-5202 (2015).

22. Kim, Y. H. et al. Multicolored organic/inorganic hybrid perovskite light-emitting diodes. Adv. Mater. 27, 1248-1254 (2015).

23. Hoye, R. L. Z. et al. Enhanced performance in fluorene-free organometal halide perovskite light-emitting diodes using tunable, low electron affinity oxide electron injectors. Adv. Mater. 27, 1414-1419 (2015).

24. Aygüler, M. F. et al. Light-emitting electrochemical cells based on hybrid lead halide perovskite nanoparticles. J. Phys. Chem. C 119, 12047-12054 (2015).

25. Yu, J. C. et al. High-performance planar perovskite optoelectronic devices: a morphological and interfacial control by polar solvent treatment. Adv. Mater. 27, 3492-3500 (2015).

26. Xing, J. et al. High-efficiency light-emitting diodes of organometal halide perovskite amorphous nanoparticles. ACS Nano 10, 6623-6630 (2016).

27. Wang, J. et al. Interfacial control toward efficient and low-voltage perovskite light-emitting diodes. Adv. Mater. 27, 2311-2316 (2015).

28. Cho, H. et al. Overcoming the electroluminescence efficiency limitations of perovskite light-emitting diodes. Science 350, 1222-1225 (2015).

29. Dou, L. et al. Atomically thin two-dimensional organic-inorganic hybrid perovskites. Science 349, 1518-1521 (2015).

30. Yuan, M. et al. Perovskite energy funnels for efficient light-emitting diodes. Nat. Nanotechnol. 11, 872-877 (2016).

31. Wang, N. et al. Perovskite light-emitting diodes based on solution-processed self-organized multiple quantum wells. Nat. Photon. 10, 699-704 (2016).

32. Xiao, Z. Efficient perovskite light-emitting diodes featuring nanometre-sized crystallites. Nat. Photonics. 11, 108-115 (2017).

33. Kulbak, M., Cahen, D. \& Hodes, G. How important is the organic part of lead halide perovskite photovoltaic cells? Efficient $\mathrm{CsPbBr}_{3}$ cells. J. Phys. Chem. Lett. 6, 2452-2456 (2015).

34. Yantara, N. et al. Inorganic halide perovskites for efficient light-emitting diodes. J. Phys. Chem. Lett. 6, 4360-4364 (2015).

35. Li, J. et al. 50 -fold EQE improvement up to $6.27 \%$ of solution-processed all-inorganic perovskite $\mathrm{Cs} \mathrm{PbBr}_{3}$ QLEDs via surface ligand density control. Adv. Mater. 29, 1603885 (2016).

36. Ling, Y. et al. Enhanced optical and electrical properties of polymer-assisted all-inorganic perovskites for light-emitting diodes. Adv. Mater. 28, 8983-8989 (2016)

37. Meng, L., You, J., Guo, T. \& Yang, Y. Recent advances in the inverted planar structure of perovskite solar cells. Acc. Chem. Res. 49, 155-165 (2016).

38. Jeon, N. J. et al. Compositional engineering of perovskite materials for high-performance solar cells. Nature 517, 476-480 (2015).

39. Veldhuis, S. A. et al. Perovskite materials for light-emitting diodes and lasers. Adv. Mater. 28, 6804-6834 (2016).

40. Zhang, W. et al. Enhanced optoelectronic quality of perovskite thin films with hypophosphorous acid for planar heterojunction solar cells. Nat. Commun. 6, 10030 (2015).

41. Small, C. E. et al. High-efficiency inverted dithienogermolethienopyrrolodione-based polymer solar cells. Nat. Photon. 6, 115-120 (2012)

42. Noh, J. H., Im, S. H., Heo, J. H., Mandal, T. N. \& Seok, S. I. Chemical management for colorful, efficient, and stable inorganic-organic hybrid nanostructured solar cells. Nano Lett. 13, 1764 (2013).

43. Shi, D. et al. Low trap-state density and long carrier diffusion in organolead trihalide perovskite single crystals. Science 347, 519-522 (2015).

44. Palma, A. L. et al. Mesoscopic perovskite light-emitting diodes. ACS Appl. Mater. Interfaces 8, 26989-26997 (2016).

45. D'Innocenzo, V. et al. Excitons versus free charges in organo-lead tri-halide perovskites. Nat. Commun. 5, 3586 (2014).

46. Dai, X. et al. Solution-processed, high-performance light-emitting diodes based on quantum dots. Nature 515, 96-99 (2014).

47. Endres, J. et al. Valence and conduction band densities of states of metal halide perovskites: a combined experimental - theoretical study. J. Phys. Chem. Lett. 7, 2722-2729 (2016).

48. Zhang, X. et al. Hybrid perovskite light-emitting diodes based on perovskite nanocrystals with organic-inorganic mixed cations. Adv. Mater 29, 1606405 (2017). 
49. Zhang, X. et al. Enhancing the brightness of cesium lead halide perovskite nanocrystal based green light-emitting devices through the interface engineering with perfluorinated monomers. Nano Lett. 16, 1415-1420 (2016).

50. Zhou, Q. et al. In situ fabrication of halide perovskite nanocrystal embedded polymer composite films with enhancednphotoluminescence for display backlights. Adv. Mater. 28, 9163-9168 (2016).

51. Li, J. et al. Single-layer halide perovskite light-emitting diodes with sub-band gap turn-on voltage and high brightness. J. Phys. Chem. Lett. 7, 4059-4066 (2016).

\section{Acknowledgements}

We thank Prof Junxi Wang and Prof Hua Yang from Institute of Semiconductors, Chinese Academy of Sciences for kindly providing GaN/InGaN LEDs to us for calibration of our measurement system, and for helping us with the CIE measurement. We also thank Prof Lu Li and Prof Xing Yang from Chongqing University of Arts and Science for brightness calibration measurement. We thank Prof Bo Wang from Beijing University of Technology for helping with contact angle measurements. We also would like to Prof Haibo Zeng from Nanjing University of Science and Technology for fruitful discussions. This work is supported by National 1,000 Young Talents awards, National Key Research and Development Program of China (Grant No. 2016YFB0700700), National Natural Science Foundation of China (Grant Numbers: 61634001, 61574133), Beijing Municipal Science \& Technology Commission (Grant No. Z151100003515004) and Young top-notch talent project of Beijing. H.T. acknowledges the Netherlands Organisation for Scientific Research (NWO) for a Rubicon grant (680-50-1511) to support his postdoctoral research at University of Toronto.

\section{Author contributions}

J.Y. conceived the idea, designed the experiment and analyzed the data. L.Z. fabricated devices and collected all data. X.Y., Q.J., P.W., Z.Y. were involved in data analysis. H.T. and M.W. carried out PLQY measurements, Y.Y. is responsible for LED response measurements. J.Y., L.Z. and X.Z. co-wrote the manuscript. H.T., B.R.S. and E.H.S improved the manuscript. J.Y. and X.Z. directed and supervised the project. All authors contributed to discussions and finalizing the manuscript.

\section{Additional information}

Supplementary Information accompanies this paper at http://www.nature.com/ naturecommunications

Competing interests: The authors declare no competing financial interests.

Reprints and permission information is available online at http://npg.nature.com/ reprintsandpermissions/

How to cite this article: Zhang, L. et al. Ultra-bright and highly efficient inorganic based perovskite light-emitting diodes. Nat. Commun. 8, 15640 doi: 10.1038/ncomms15640 (2017).

Publisher's note: Springer Nature remains neutral with regard to jurisdictional claims in published maps and institutional affiliations.

\section{(c) (1)}

This work is licensed under a Creative Commons Attribution 4.0 International License. The images or other third party material in this article are included in the article's Creative Commons license, unless indicated otherwise in the credit line; if the material is not included under the Creative Commons license, users will need to obtain permission from the license holder to reproduce the material. To view a copy of this license, visit http://creativecommons.org/licenses/by/4.0/

(C) The Author(s) 2017 